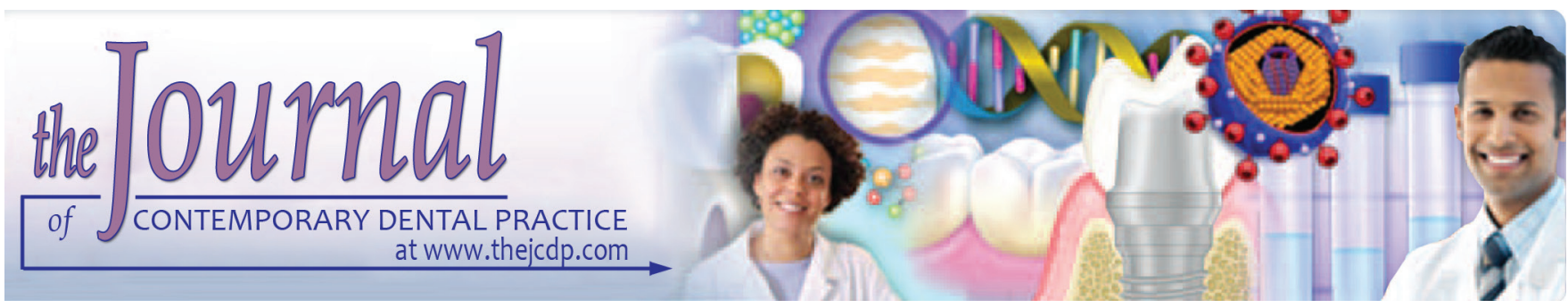

\title{
Evaluating Effectiveness of Axiostat Hemostatic Material in achieving Hemostasis and Healing of Extraction Wounds in Patients on Oral Antiplatelet Drugs
}

\footnotetext{
${ }^{1}$ Saurabh Sharma, ${ }^{2}$ Tejraj P Kale, ${ }^{3}$ Lingaraj J Balihallimath, ${ }^{4}$ Abhishek Motimath
}

\begin{abstract}
Aim: The aim of this study was to evaluate the efficacy of Axiostat Hemostatic Dental dressing in achieving hemostasis postextraction and determining its effect on pain and healing of the extraction wound, compared with control, i.e., conventional method of extraction in patients on oral antiplatelet therapy.
\end{abstract}

Materials and methods: Totally, 40 patients on oral antiplatelet drugs were included in the study and overall 80 extractions were done applying split mouth study design, without altering patient's drug regime. Extraction sites were divided into two groups: Group I received Axiostat Hemostatic Dental Dressing (study site), and group II received conventional method; pressure pack with sterile gauze under biting pressure followed by suturing if required (control site) was used to attain hemostasis.

Results: Extraction sites treated with Axiostat Hemostatic Dressing achieved hemostasis earlier (mean 1 minute 13 seconds) compared with control sites (mean $=14$ minutes 1 second), which was also statistically significant $(p<0.001)$. Postoperative pain was considerably lower and significantly better healing was seen in the study group $(p<0.001)$ compared with the control.

Conclusion: Axiostat demonstrated to be an effective hemostatic agent that considerably lessens the bleeding time in patients on oral antiplatelet drugs postextraction. In addition, it even offered minimal postoperative pain and improved healing of the extraction wound. On comparing the results of this study with our study on HemCon Dental Dressing, Axiostat Dental Dressing (ADD) is found to be as effective and at par in achieving hemostasis in patients on oral antiplatelet therapy.

Clinical significance: The past few decades have seen an upsurge in use of low-dose aspirin either alone or in combination with other drugs. When these patients require dental/

${ }^{1-4}$ Department of Oral and Maxillofacial Surgery, KLE VK Institute of Dental Sciences, Belgaum, Karnataka, India

Corresponding Author: Saurabh Sharma, Department of Oral and Maxillofacial Surgery, KLE VK Institute of Dental Sciences Belgaum, Karnataka, India, Phone: +919972789290, e-mail: doc.saurabh@outlook.com maxillofacial treatment, earlier concept of stopping these medications is associated with increased risk of thromboembolic event. The present study highlights an alternative approach using ADD which aids in quick hemostasis, accentuates healing, and reduce postoperative pain.

Keywords: Antiplatelet, Axiostat, Chitosan, Extraction, Hemorrhage, Wound healing.

How to cite this article: Sharma S, Kale TP, Balihallimath LJ, Motimath A. Evaluating Effectiveness of Axiostat Hemostatic Material in achieving Hemostasis and Healing of Extraction Wounds in Patients on Oral Antiplatelet Drugs. J Contemp Dent Pract 2017;18(9):802-806.

Source of support: Nil

Conflict of interest: None

\section{INTRODUCTION}

Outpatient dental surgery in patients with hemostatic alterations has become a common practiceover the past decade demanding a specific approach by the dentist and an interdisciplinary interaction with various health care professionals. ${ }^{1-3}$ Extraction in patients on oral antiplatelet drugs is one of the most common complications confronted today, making them a challenge to treat.

Despite the numerous benefits of antiplatelet drugs they are not without risk, i.e., tendency to bleed excessively. ${ }^{4,5}$

According to Nichols and Bowie, ${ }^{6}$ frequency of patients on antiplatelet therapy seeking dental treatment is increasing day by day worldwide and with the availability of medical facilities it is going to increase further. The use of international normalized ratio (INR) is the gold standard for monitoring patients on antiplatelet therapy, recommended by the American Heart Association and the British Society for Hematology.

Some authors recommend managing these patients by temporarily discontinuing the drug therapy, although literature shows a small but significant risk of untoward 
complications that might be associated with long-term morbidity, 7,8 while others recommend the use of local measures to stop bleeding, which are simple to use but usually not effective per se.

Axiostat Dental Dressing (Axiostat Bio-solutions, Gujarat, India) offers a new way to achieve hemostasis. It is a highly electropositive sponge-like biomaterial extracted from crustaceans, having hemostatic potential that acts by attracting negatively charged red blood cells (RBCs) and platelets, thus forming a strong seal at the extraction wound. ${ }^{9,10}$ The hemostatic action of Axiostat Dental Dressing is not solely dependent on the clotting cascade, but it acts as a scaffold and provides primary seal, allowing the body to initiate its coagulation pathway effectively. ${ }^{9}$ Clot formation is aided by the strong sealing action which in turn offersan antibacterial barrier over the extraction wound.

The objective of this prospective study is to evaluate the efficacy of Axiostat Hemostatic Dental Dressing in controlling postextraction bleeding diathesis, and determining its effects on pain and healing of the extraction wound as compared with conventional pressure dressings used in patientson oral antiplatelet therapy.

\section{MATERIALS AND METHODS}

In this prospective clinical study, 40 patients on oral antiplatelet drugs were included and 80 extractions were done applying split mouth study design, without altering patient's drug regime. The institutional review board and ethics committee approval was taken for the study design and selection of patients. Management of extraction sites in each patient were divided into two groups: Group I received Axiostat Hemostatic Dental Dressing (study group), and group II received conventional method; pressure pack with sterile gauze under biting pressure followed by suturing if required (control group) was used to attain hemostasis.

Inclusion criteria were patients undergoing multiple tooth extractions aged between 35 and 70 years with INR values $\leq 3.5$ (1-3.5) and if present, sugar level under control. Exclusion criteria were patients undergoing a single tooth or multiple extractions in same quadrant; and those with anallergy to seafood.

Antiplatelet medications are mainly indicated for prevention and treatment of thrombotic events in patients with certain medical conditions, such as heart attack, pulmonary embolism, stroke with or without atrial fibrillation, any heart surgery (especially prosthetic replacement heart valve), coronary heart disease, such as stable angina, unstable angina and heart attack, patients with coronary stent, peripheral vascular disease/peripheral arterial disease, and apical/ventricular/mural thrombus.
Each patient underwent an assessment of general medical status and hematological investigations preoperatively including hemoglobin estimation, INR, platelet count, bleeding and clotting time,and X-ray if required. Similar extraction sites were selected within each patient, i.e., sites withcomparable socket size. To reduce the bias and study variability, similar contralateral teeth were extracted wherever possible (33 patients), in remaining 7 patients teeth with similar socket size were included (i.e., single rooted tooth for single rooted tooth and molar for molar).

Random selection of the operative site was done for study and control group in different quadrants. Preventive antibiotic prophylaxis was given to the patients, if indicated 1 hour before extraction with $2 \mathrm{gm}$ amoxicillin orally. Each patient underwent minimally traumatic extractions under local anesthesia using plain lignocaine, by a single surgeon. Axiostat Dressing was loosely fitted into the extraction socket, at the height of crestal bone, under finger pressure for 30 seconds and upon achieving hemostasis, it was removed by irrigating with saline. In the control site, sutures were placed whenever hemostasis was not achieved under biting pressure over sterile gauze piece after 900 seconds (15 minutes). Time to achieve hemostasis was noted for both study and control sites using a stopwatch. Patients were given a list of routine postoperative instructions and the contact number of the surgeon who could be contacted in case of postoperative bleeding.

All patients were prescribed:

- Tablet diclomol (Diclofenac sodium $50 \mathrm{mg}+$ paracetamol $500 \mathrm{mg}$ ) every 8 hours for 3 days

- Capsule amoxicillin 500 mg every 8 hours for 3 days Patients were reviewed by another surgeon on 7 th postoperative day for assessment of pain and healing, using visual analog pain score chart and soft tissue healing index by Landry et al. ${ }^{11,12}$ Secondary bleeding was assessed by the presence or absence of liver clots and patients self-reporting. Statistical analysis was done using Mann-Whitney U-test.

\section{RESULTS}

Of the 40 patients, there were 29 males and 11 females and their age ranged from 44 to 70 years.

The most common systemic diseases we came across in patients were thrombosis, valvopathy, followed by cardiopathy.

Time to achieve hemostasis was recorded for both study and control sites using stopwatch. Mean and standard deviation (SD) was calculated.

Study group achieved hemostasis in a mean of 1 minute 13 seconds, compared with control which took a mean of 14 minutes 1 second, which was statistically 


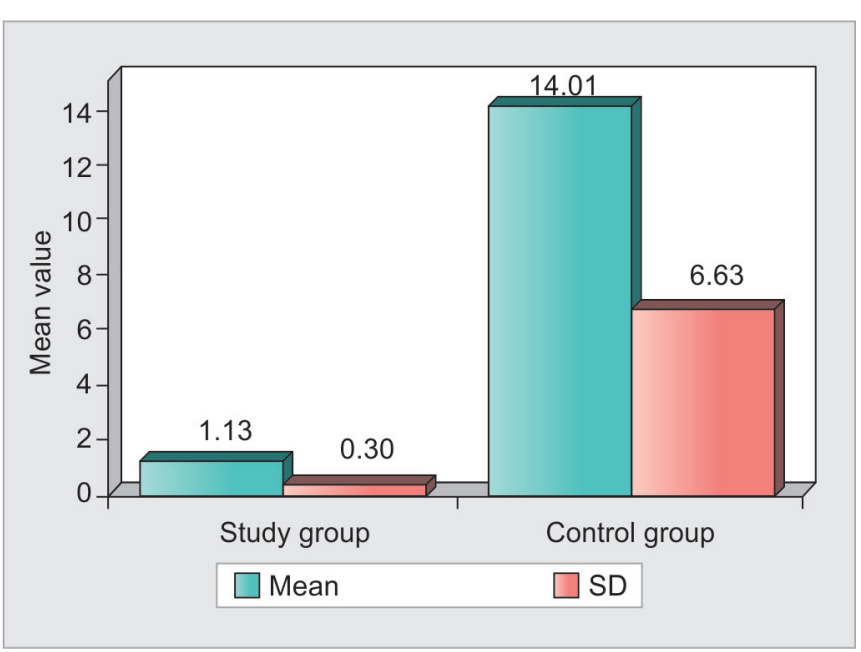

Graph 1: Comparison of study and control groups with bleeding time in minutes

significant $(\mathrm{p}<0.001)$, demonstrating improved hemostasis with the use of Axiostat Hemostatic Dressing (Graph 1).

Pain experienced by the patients throughout the week during daily activities was documented on 7th postoperative day, with the visual analog scale in a range of 0 to 10 , with 0 depicting no pain and 10 depicting worst pain experienced by the patient. ${ }^{11}$ Average pain score in study group (1.93) was considerably lower than in the control group (3.63) and is also statistically significant $(\mathrm{p}<0.001)($ Graph 2).

There was no postextraction complication perceived by the surgeon on the 7th day postoperatively.

Healing was significantly better in study group compared with the control in terms of better epithelialization, tissue color, and response to palpation, assessed using soft tissue healing indexby Landry et al. ${ }^{12}$ (Graph 3).

In this study, we treated 40 patients with INR values ranging from 1.09 to 3.15 and no significant bleeding complications were reported except for one in a 68-yearold patient with an INR of 3.15, prolonged bleeding was

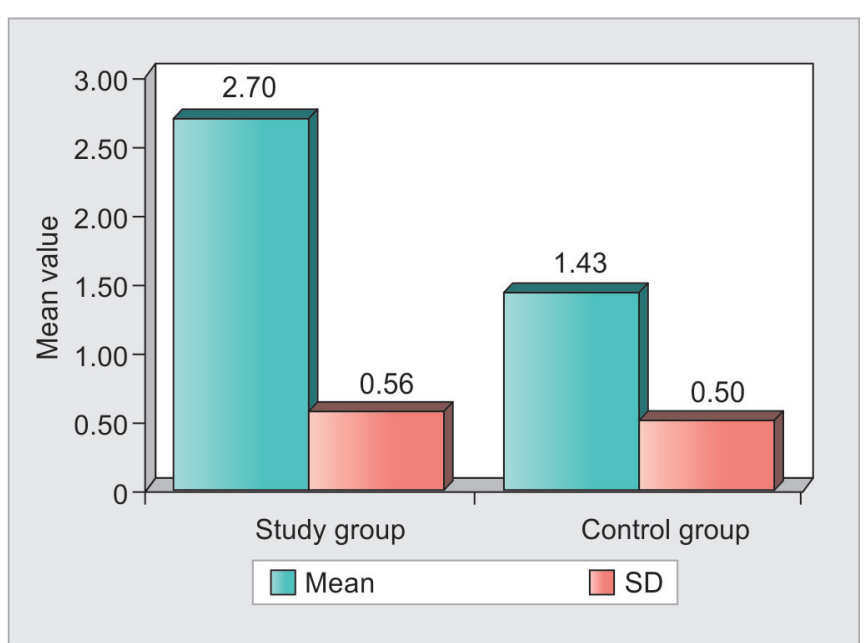

Graph 3: Comparison of study and control groups with healing scores

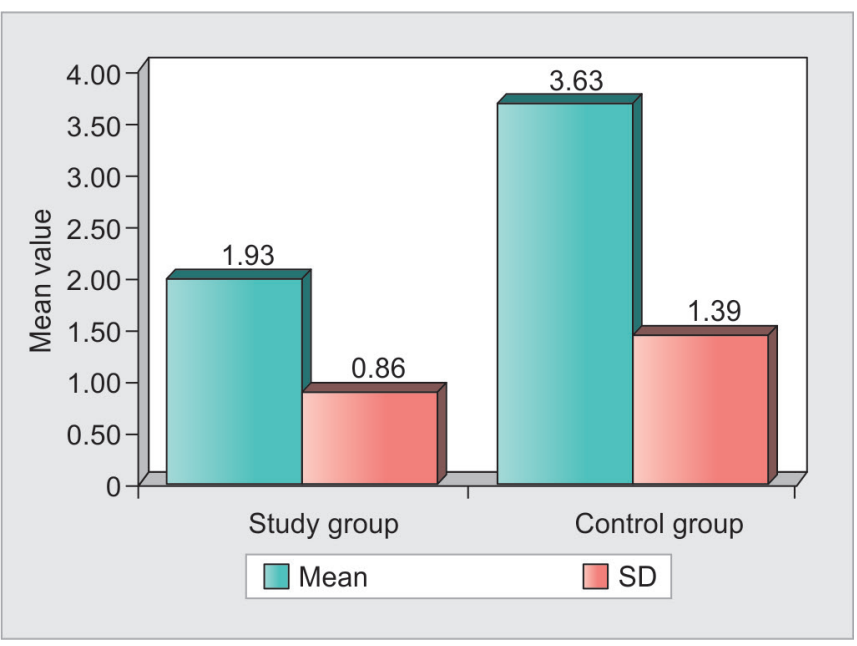

Graph 2: Comparison of study and control groups with pain scores

evident from the control site 6 hours postextraction. However, it was controlled by compression with sterile gauze and placing sutures later. Remaining patients showed complete hemostasis postextraction. None of the patients had any delayed bleeding on reviewing 7 days postoperatively nor required admission to the hospital at any stage.

There was no evidence of postoperative trismus due to bleeding at local anesthetic injection sites nor was there any incidence of any untoward event, in any of the patients.

\section{DISCUSSION}

Patients with hemorrhagic diathesis have tendency to bleed excessively postextraction, which may increase the risk of infection and also cause delayed wound healing. ${ }^{13}$ Planning dental extractions in them presents a dilemma. The INR is now the gold standard for assessing these patients before extraction. ${ }^{14,15}$ Oral antiplatelet drugs, such as aspirin and clopidogrel, irreversibly inhibit platelet aggregation and this lasts for the life of the platelets (7-10 days), leading to greater bleeding tendency and increase in INR values in patients taking them. ${ }^{16}$ The effect is only overcome by the manufacture of new platelets. Lippert and Gutschik ${ }^{17}$ in their study recommended that INR should not be higher than 4.0, if patient is to be taken for dental procedure. ${ }^{18}$ In our study, we achieved hemostasis in a patient with INR 3.15 using ADD (1.20 minutes), compared with conventional method (22.5 minutes), thus demonstrating the effectiveness of using ADD in patients on antiplatelet drugs (Graph 4), even when INR is on the higher side.

Correlation between INR and bleeding time in both study and control groups is shown in Graph 5 and Figure 1. The control group showed increase in bleeding time with concomitant increase in INR values, whereas in the study 


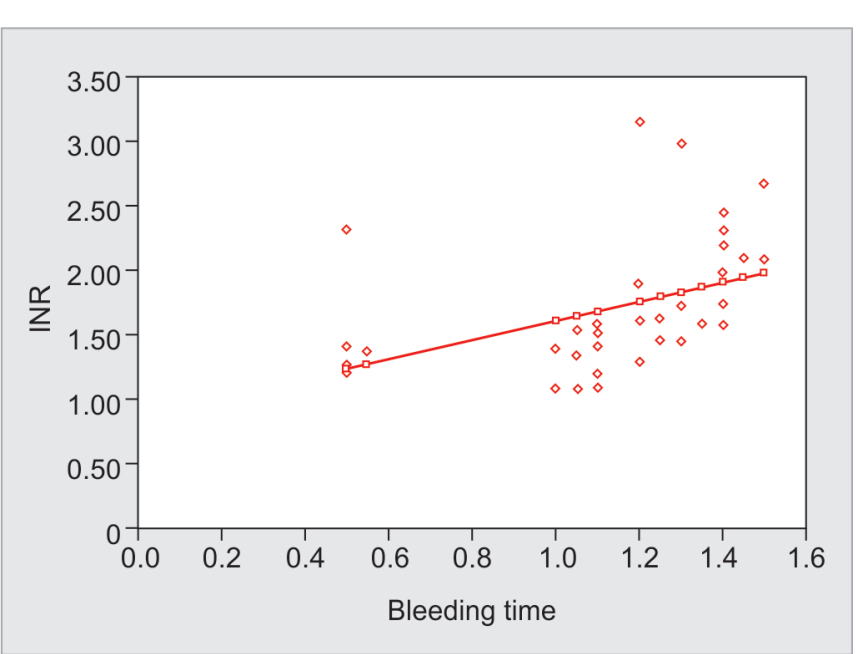

Graph 4: Correlation between INR and bleeding time in study group (ADD)

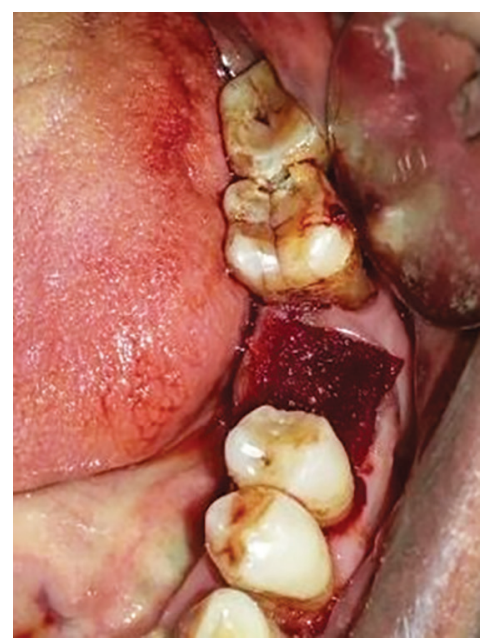

Fig. 1: Axiostat dressing in situ

group postextraction bleeding was controlled earlier using Axiostat Dental Dressing.

Although conventional agents are effective in controlling postextraction bleeding, they do not hold well in every situation. In our study, resultant time to achieve hemostasis was shorter when Axiostat Dressing was used compared with conventional method of pressure pack in all the patients,highlighting the efficacy of ADD in achieving hemostasis earlier. The early hemostatic action of Axiostat Dental Dressing is by providing stable scaffold for RBCs to bind and aggregate under electrostatic attraction, and also to its adaptation within the socket providing frictional lock, under finger pressure. ${ }^{10}$

The ADD is a chitosan-based material, which has got cell adhesion potential and is consistent according to Cunha-Reis et al. ${ }^{17}$ Shen et $\mathrm{al}^{19}$ in their study stated that growth factors are released from human platelets stimulated by chitosan exposure, which can explain better healing observed with Axiostat Dressing. ${ }^{20}$ Klokkevold et $\mathrm{al}^{21}$ in their study stated that chitosan potentiates the

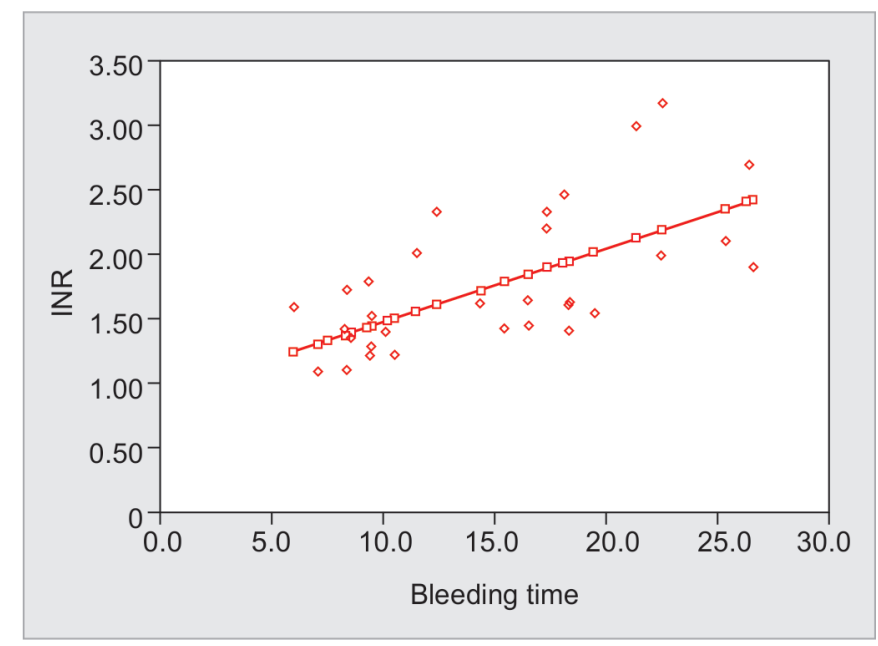

Graph 5: Correlation between INR and bleeding time in control group

differentiation of osteoprogenitor cells and may facilitate the formation of bone and help in healing, which can be attributed to superior healing in the study group.

The antibacterial property of chitosan (Axiostat) providing a barrier against wide range of oral microbes and early hemostasis could be the reason behind minimal pain and complications in the study group compared with the control and also for superior postoperative healing in study group. ${ }^{21,22}$

Comparing the results of this study with our previous study on HemCon Dental Dressing (HDD), the mean time taken to achieve hemostasis is significantly lower when ADD (73 seconds) or HDD (53 seconds) was used compared with conventional method. ${ }^{4}$ Increase in the time to achieve hemostasis using ADD is attributed to the fact that the patients with INR up to 3.5 were included in this study in contrast to our previous study on Hemcon dressing where INR limit was 3. On comparing the results of postoperative healing and pain between ADD and Hemcon dressing, both showed similar potential of superior healing and minimal postextraction pain and complications.

It is imperative to evaluate the risks of transoperative and postoperative hemorrhage, as well as the extent of surgical trauma subjected to the patient to establish an adequate protocol.

Therefore, we recommend that performing a surgical procedure as atraumatic as possible, strict observance of all surgical steps, and patient's compliance with the postoperative instructions are primordial factors to be considered at all times in all the patients, especially those on antiplatelet therapy. ${ }^{23}$ The results of this study could validate the use of Axiostat Dental Dressing postextraction for preventing postextraction bleeding in patients on oral antiplatelet drugs as well aspatients with increased bleeding tendency. 


\section{CONCLUSION}

Extractions in patients on oral antiplatelet therapy can be safely performed while achieving effective hemostasis with the help of Axiostat Dental Dressing. Results show that Axiostat is alocally available, new-generation hemostatic agent which helps in achieving early hemostasis postextraction, along with better healing and less postoperative pain. On comparing the results of this study with our previous study on HDD, Axiostat Dental Dressing is found to be as effective and at par in achieving hemostasis in patients on oral antiplatelet therapy. ${ }^{4}$

\section{REFERENCES}

1. Hirsh J, Dalen JE, Deykin D, Poller L. Oral anticoagulants: Mechanisms of action, clinical effectiveness, and optimal therapeutic range. Chest 1992 Oct;102(4 Suppl):312S-326S.

2. Davies, JA.; Tuddenham, EG. Haemostasis and thrombosis. In: Weatherall DJ, Ledingham JG, Warrell DA, editors. Oxford textbook of medicine. 2nd ed., Vol. 19. Oxford: Oxford Medical Publications; 1988. p. 228-229.

3. Weinmann EE, Salzman EW. Deep-vein thrombosis. N Engl J Med 1994 Dec;331(24):1630-1641.

4. Kale TP, Singh AK, Kotrashetti SM, Kapoor A. Effectiveness of HemCon Dental Dressing versus conventional method of haemostasis in 40 patients on oral antiplatelet drugs. Sultan Qaboos Univ Med J 2012 Aug;12(3):330-335.

5. Yacabucci JE, Kramer HS Jr. Platelet defects of importance in oral surgery. J Oral Surg 1972 Jul;30(7):478-485.

6. Nichols WL, Bowie EJ. Standardization of the prothrombin time for monitoring orally administered anticoagulant therapy with use of the international normalized ratio system. Mayo Clin Proc 1993 Sep;68(9):897-898.

7. Longstreth WT Jr, Bernick C, Fitzpatrick A, Cushman M, Knepper L, Lima J, Furberg CD. Frequency and predictors of stroke death in 5,888 participants in the Cardiovascular Health Study. Neurology 2001 Feb;56(3):368-375.

8. Biondi-Zoccai GG, Lotrionte M,Agostoni P,Abbate A, Fusaro M, Burzotta F, Testa L, Sheiban I, Sangiorgi G. A systematic review and meta-analysis on the hazards of discontinuing or not adhering to aspirin among 50,279 patients at risk for coronary artery disease. Eur Heart J 2006 Nov;27(22):2667-2674.

9. Malmquist JP, Clemens SC, Oien HJ, Wilson SL. Hemostasis of oral surgery wounds with the HemCon Dental Dressing. J Oral Maxillofac Surg 2008 Jun;66(6):1177-1183.
10. Wedmore I, McManus JG, Pusateri AE, Holcomb JB. A special report on the chitosan-based hemostatic dressing: experience in current combat operations. J Trauma 2006 Mar;60(3):655-658.

11. Campbell WI, Lewis S. Visual analogue measurement of pain. Ulster Med J 1990 Oct;59(2):149-154.

12. Landry RG, Turnbull RS, Howley T. Effectiveness of benzydamyne $\mathrm{HCl}$ in the treatment of periodontal post-surgical patients. Research in Clinic Forums. 1988;10:105-118.

13. Ercetin S, Haznedaroglu IC, Kurt M, Onal IK, Aktas A, Kekilli M. Safety and efficacy of Ankaferd blood stopper in dental surgery and bleeding. Oral Surg Oral Med Oral Pathol Oral Radiol Endod 2010;1:1-5.

14. Morrison M, Caldwell A, McQuaker G, Fitzsimons EJ. Discrepant INR values: a comparison between Manchester and Thrombotest reagents using capillary and venous samples. Clin Lab Haematol 1989 Dec;11(4):393-398.

15. Bussey HI, Force RW, Bianco TM, Leonard AD. Reliance on prothrombin time ratios causes significant errors in anticoagulation therapy. Arch Intern Med 1992 Feb;152(2):278-282.

16. Poller L, Thomson JM, Taberner DA, Clarke DK. The correction of coagulometer effects on international normalized ratios: a multicentre evaluation. Br J Haematol 1994 Jan;86(1):112-117.

17. Lippert $\mathrm{S}$, Influence of porosity and fibre diameter on the degradation of chitosan fibre-mesh scaffolds and cell adhesion. J Mater Sci Mater Med 2007 Feb;18(2):195-200.

18. CAPRIE Steering Committee. A randomised, blinded, trial of clopidogrel versus aspirin in patients at risk of ischaemic events (CAPRIE). CAPRIE Steering Committee. Lancet 1996 Nov;348(9038):1329-1339.

19. Shen EC, Chou TC, Gau CH, Tu HP, Chen YT, Fu E. Releasing growth factors from activated human platelets after chitosan stimulation: a possible bio-material for platelet-rich plasma preparation. Clin Oral Implants Res 2006 Oct;17(5):572-578.

20. Della Valle A, Sammartino G, Marenzi G, Tia M, Espedito di Lauro A, Ferrari F, Lo Muzio L. Prevention of postoperative bleeding in anti-coagulated patients undergoing oral surgery: use of platelet-rich plasma gel. J Oral MaxillofacSurg 2003 Nov;61(11):1275-1278.

21. Klokkevold PR, Vandemark L, Kenney EB, Bernard GW. Osteogenesis enhanced by chitosan (poly-N-acetyl glucosaminoglycan) in vitro. J Periodontol 1996 Nov;67(11):1170-1175.

22. Burkatovskaya M, Tegos GP, Swietlik E, Demidova TN, PCastano A, Hamblin MR. Use of chitosan bandage to prevent fatal infections developing from highly contaminated wounds in mice. Biomaterials 2006 Aug;27(22):4157-4164.

23. Speechley JA, Rugman FP. Some problems with anticoagulants in dental surgery. Dent Update 1992 Jun;19(5):204-206. 